\title{
Estudo Comparativo do Nível de Velocidade de Jovens de Escolas Públicas de Barbalha e de Juazeiro do Norte - Ceará
}

\author{
Thiago Cabral Souza ${ }^{1}$; João David Alan Santos ${ }^{2}$; Paulo Geovane Bezerra de Sousa ${ }^{3}$
}

\begin{abstract}
Resumo: As vantagens da prática diária de atividade física sobre a saúde estão bem evidenciadas na literatura científica e medicina do esporte. O objetivo deste estudo foi investigar e comparar o nível de aptidão física relacionada ao nível de velocidade dos escolares de onze a quatorze anos, de uma escola pública no município de Barbalha uma escola pública do município de Juazeiro do Norte - Ce. O estudo de campo caracterizou-se como do tipo transversal, descritivo e quantitativo. A amostra foi constituída de 100 crianças de ambos os sexos, com idades entre 11 e 17 anos, que não praticam regularmente atividades desportivas, apenas uma vez por semana na aula de Educação Física de suas escolas. Foram utilizados os protocolos de baterias de testes do Projeto Esporte Brasil. A variável de aptidão física medida foi à velocidade. Os resultados assinalam que os meninos têm melhor desempenho médio que as meninas, até em função da força que os meninos geneticamente têm mais que as garotas, os tornando-os através dessa valência os melhores resultados em sua média geral. $\mathrm{O}$ estudo concluiu que os meninos de fato são mais velozes que as meninas e que existe variação de escola para escola.
\end{abstract}

Palavras-Chave: Escolares. Aptidão Física. Velocidade.

\section{Comparative Study of the Velocity Level of Young Students from Public Schools of Barbalha and Juazeiro do Norte, in the state of Ceará}

\begin{abstract}
The benefits of daily physical activity on health are well evidenced in the literature , and sports medicine. The aim of this study was to investigate and compare the level of physical fitness related to the speed level of the students from eleven to fourteen years in a public school in the city of Barbalha a public school in the city of Juazeiro do Norte - Ce . The field study was characterized as the cross-sectional descriptive and quantitative type. The sample consisted of 100 children of both sexes, aged between 11 and 17 years, who do not regularly engage in sports activities, only once a week in the Physical Education class in their schools . Protocols batteries of tests for Sport Project Brazil were used. The variable measuring physical fitness was the speed . The results indicate that boys have better average performance than girls, according to the power that genetically have more boys than girls, making them the valence through that the best results in your overall average. The study found that boys really are faster than girls and that there is variation from school to school .
\end{abstract}

Keywords : School . Physical Fitness . Speed.

\footnotetext{
${ }^{1}$ Graduação em Educação Física na Universidade Leão Sampaio - UNILEÃO. E-mail: cabral.10@ gmail.com; ${ }^{2}$ Docente orientador do Curso de Licenciatura em Educação Física da Universidade Leão Sampaio - UNILEÃO. E-mail: jalan.david10@gmail.com

${ }^{3}$ Graduação em odontologia pela Universidade Federal da Paraíba - UFPB. E-mail: pgodonto@ hotmail.com.
} 
Id on Line Revista Multidisciplinar e de Psicologia

Id on Line Multidisciplinary and Psycology Journal

\section{Introdução}

A Educação Física escolar vem desempenhando um papel importante na sociedade e mostrando seu desenvolvimento, de forma a contribuir para a saúde da população, em especial, dos estudantes. Observamos que as valências físicas relacionadas à saúde (composição corporal, força e resistência muscular, aptidão cardiorrespiratória e a flexibilidade) são de suma importância e estão presentes na aula de Educação física. (SANTOS, ROBERTO, 2010)

A prática dessas atividades física estimula os escolares a optar por uma melhor qualidade de vida, sendo possível a prevenção de doenças cardiovasculares. A velocidade é uma das capacidades mais importantes exigidas nos desportos, consiste na capacidade de mover-se rapidamente. O seu desenvolvimento é de extrema importância, pois esta se apresenta em vários tipos de esportes, de diferentes maneiras. (PAULO, BISOTTO, 2013)

Sendo uma valência física muito importante que se destaca nos desportos coletivos e individuais podendo ser definida como a capacidade de realizar movimentos de forma mais rápida possível. (OLIVEIRA et al., 2010) define que velocidade é a habilidade de se movimentar ou deslocar com rapidez. Cristina (2003) acrescenta que a velocidade não é simplesmente poder realizar movimentos rápidos, mas também coordenar estes movimentos.

Melo et al (2009) melhorando a capacidade de força do individuo ele futuramente poderá adquiri também uma maior velocidade. Sendo assim a velocidade do indivíduo esta diretamente ligada à força. $\mathrm{O}$ treinamento de força é uma variável que deve ser especificamente trabalhada para cada modalidade esportiva. Este estudo te com objetivo comparar os níveis de aptidão física relacionada a o nível de velocidade em duas escolas de sistema e públicos diferentes em duas cidades do cariri cearense.

\section{Metodologia}

O presente estudo de campo transversal caracteriza-se como descritivo e quantitativo. A população do estudo são crianças e adolescentes de duas escolas públicas de duas cidades do cariri cearense. $\mathrm{Na}$ amostra do estudo participaram do escolares de ambos os sexos, de uma escola pública localizada no município de Barbalha - Ce, e uma escola pública do município de 
Id on Line Revista Multidisciplinar e de Psicologia

Id on Line Multidisciplinary and Psycology Journal

Juazeiro do Norte - Ce, sendo 110 alunos de cada instituição, totalizando 220 avaliados com idades entre 11 e 17 anos, ficando 46 meninas e 56 meninos para cada escola, estando estes matriculados do $6^{\circ}$ ao $9^{\circ}$ ano do ensino fundamental. A amostra foi composta pelo critério de aleatoriedade.

Foi feita a comparação dos níveis de velocidade utilizando o teste de $2 \mathrm{~m}$ mestre da bateria de teste da PROESP-BR (GAY, 2012) seguindo o seguinte protocolo na quadra foi demarcada com três linhas paralelas no solo distribuído da seguinte forma: a primeira (linha de partida); a segunda, distante $20 \mathrm{~m}$ da primeira (linha de cronometragem) e a terceira linha, marcada a um metro da segunda (linha de chegada). A terceira linha serve como referência de chegada para o aluno na tentativa de evitar que ele inicie a desaceleração antes de cruzar a linha de cronometragem. Dois cones para a sinalização da primeira e terceira linhas e o tempo foi registrado através de um cronometro em segundos.

Sendo aplicado um aquecimento antes do teste, seguindo a sugestão do PROESP-BR. Ao inicio o estudante parte da posição de pé, com um pé avançado à frente imediatamente atrás da primeira linha e será informado que deverá cruzar a terceira linha o mais rápido possível. Ao sinal do avaliador, o aluno deverá deslocar-se, o mais veloz possível, em direção à linha de chegada. $\mathrm{O}$ cronometrista deverá acionar o cronômetro no momento em que o avaliado der o primeiro passo (tocar ao solo), ultrapassando a linha de partida. Quando o aluno cruzar a segunda linha (dos 20 metros) será interrompido o cronômetro. O cronometrista registrará o tempo do percurso em segundos e centésimos de segundos (duas casas após a vírgula).

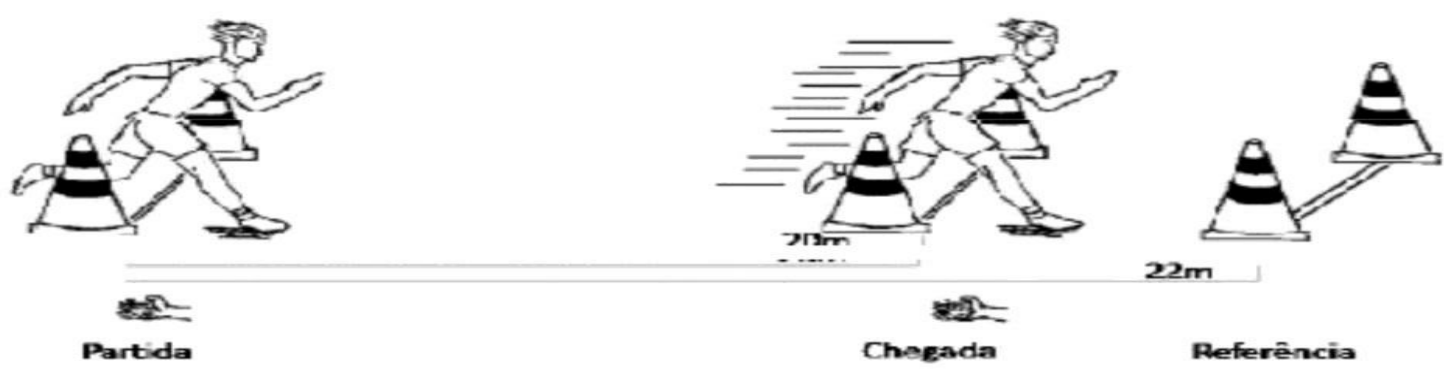

Figura 1: Teste de 20 metros 


\section{Resultados e Discussões}

Foi analisado a velocidade média do teste de 20 metros em função das escolas e do sexo dos participantes, os efeitos obtidos são que os alunos do sexo masculino da escola do município de Juazeiro do Norte-Ce têm uma velocidade média maior que os alunos do sexo feminino.

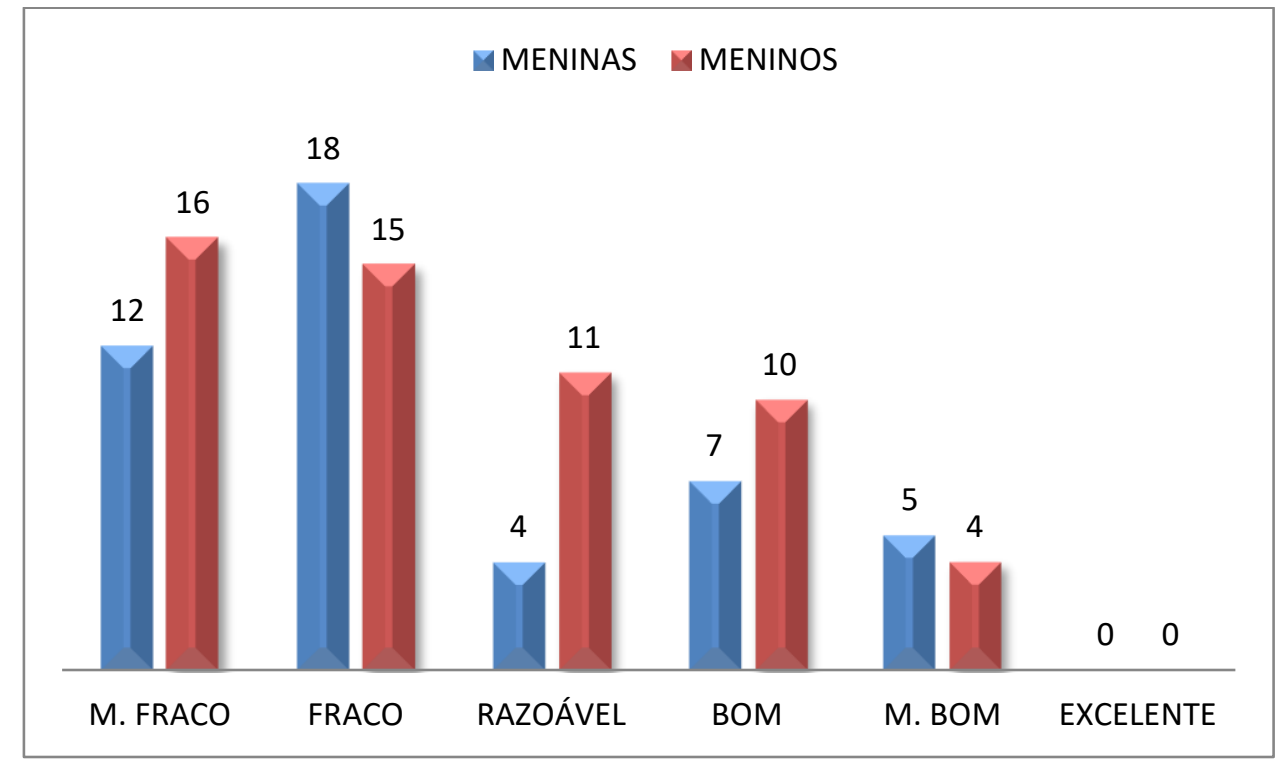

Gráfico 1: Resultado do teste de velocidade entre gêneros da escola de Juazeiro do Norte-CE

Já os resultados dos alunos do município de Barbalha-ce foram o inverso, se da que os alunos do sexo feminino têm uma velocidade média maior que os alunos do sexo masculino alunos do sexo masculino. 


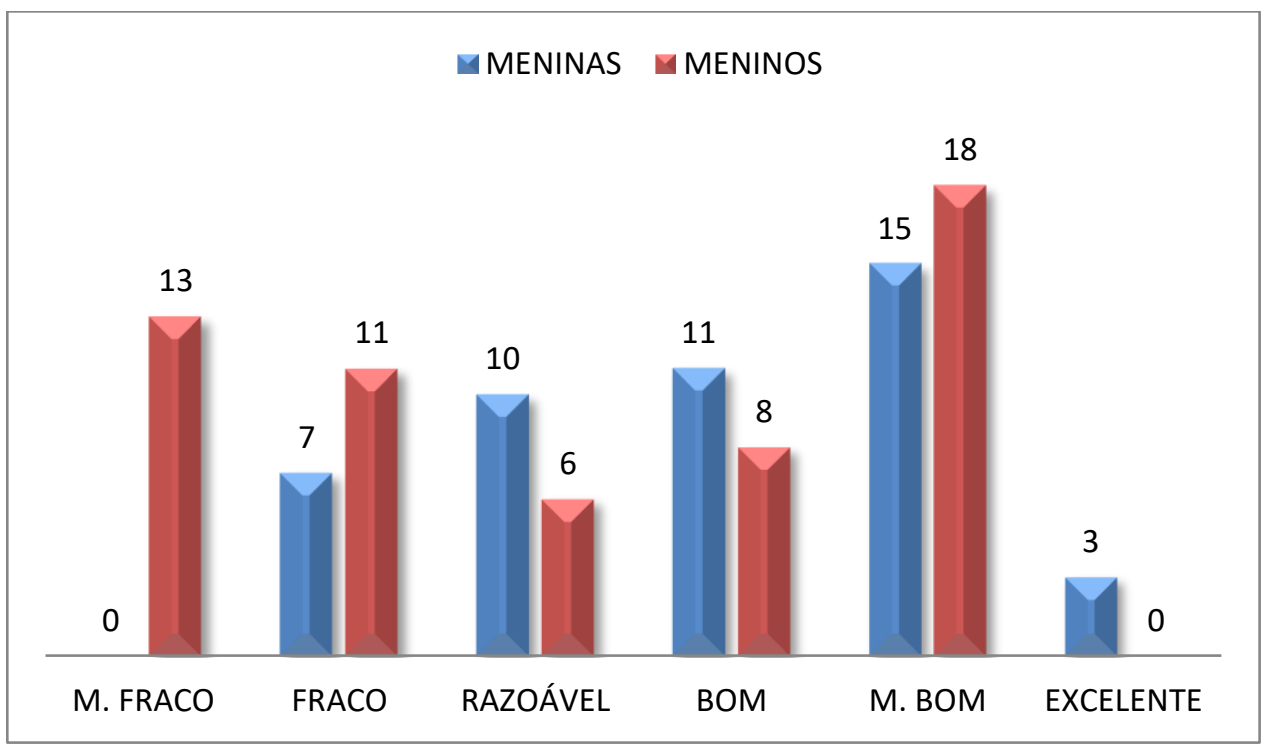

Gráfico 2: Resultado do teste de velocidade entre gêneros da escola de Barbalha-CE

Comparando-se os testes de uma escola com a outra, foi comprovado que à escola do município de Barbalha-Ce obteve o melhor resultado de ambos os sexos. Desconsiderando a idade dos analisados os meninos da escola do município de Juazeiro do Norte-Ce apresentaram a média geral de 3,99m/s de velocidade, já as meninas apresentaram velocidade média de 4,41 $\mathrm{m} / \mathrm{s}$. Na escola do município de Barbalha-Ce obtivemos as seguintes médias gerais de velocidade meninos $3,70 \mathrm{~m} / \mathrm{s}$ e meninas $3,79 \mathrm{~m} / \mathrm{s}$.

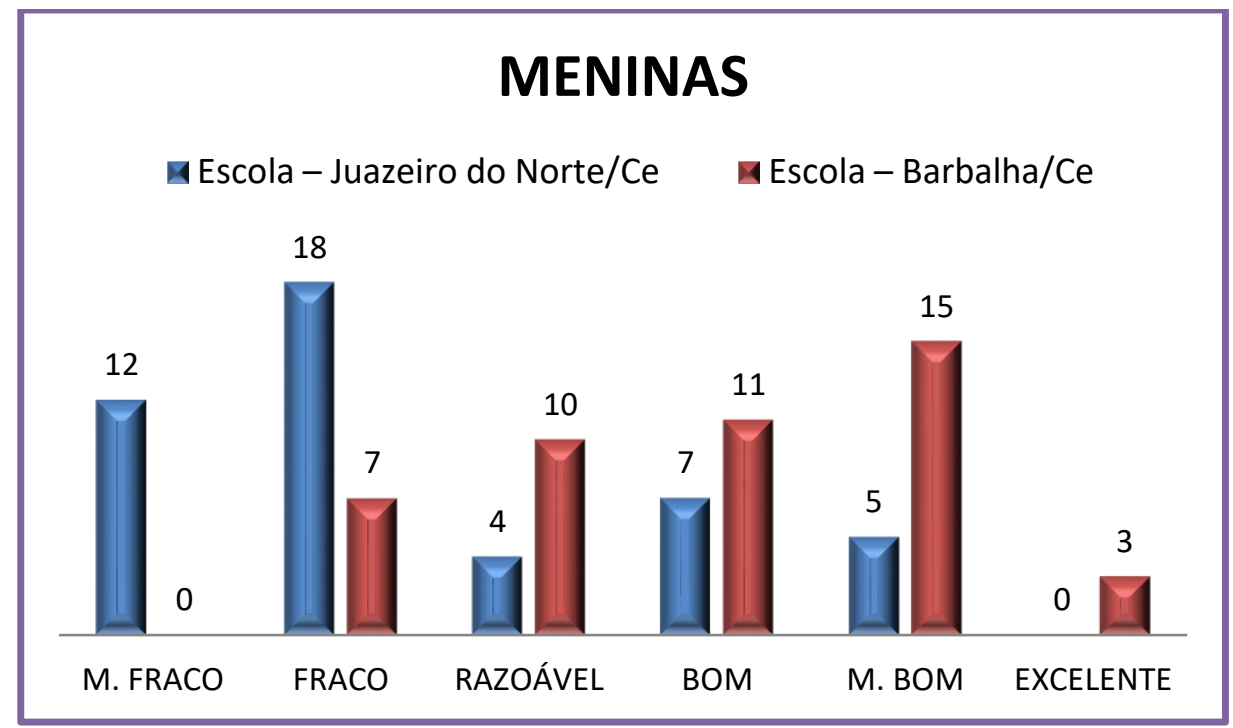

Gráfico 3: Comparação entre o sexo feminos das escolas. 


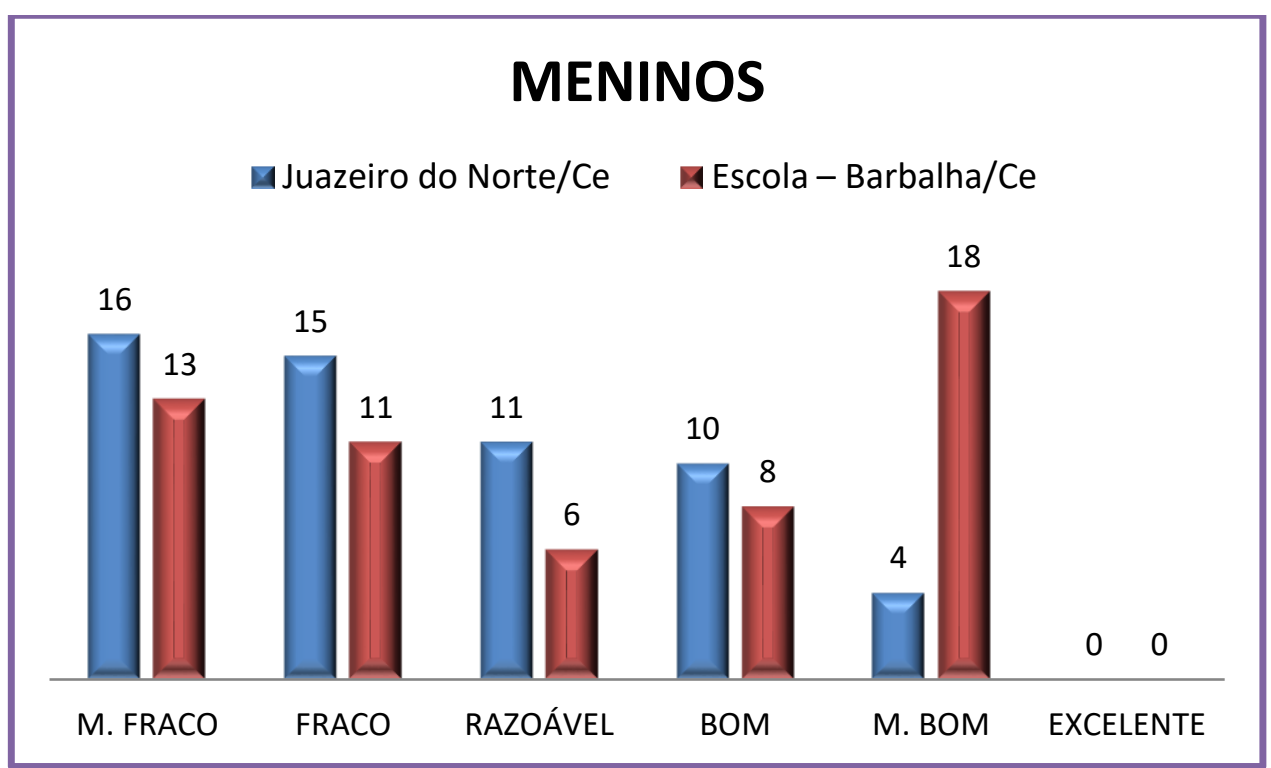

Gráfico 4: Comparação entre o sexo masculino das escolas.

Por meio desta pesquisa podemos perceber que, o padrão de velocidade geral analisado foi que os meninos conseguiram uma velocidade média de aproximadamente $3,99 \mathrm{~m} / \mathrm{s}$, e as meninas uma média geral de 4,10 m/s. Procedidos estes que confirmam a hipótese de que os indivíduos do sexo masculino seriam mais rápidos que os indivíduos do sexo oposto.

\section{TABELA 1 - MÉdia geral de gÊNERO E ESCOLAS}

\begin{tabular}{cccc} 
& ESCOLA JUAZEIRO & ESCOLA BARBALHA & MÉDIAS GERAIS \\
& DO NORTE & & ESCOLAS \\
FEMININO & 4,41 & 3,79 & 4,10 \\
MASCULINO & 3,99 & 3,70 & 3,85 \\
\hline
\end{tabular}

Para Oliveira (2010) apud Letzelter (1979), os sujeitos do sexo feminino têm uma velocidade menor que os sujeitos do sexo masculino devido aos meninos serem geneticamente mais fortes que as meninas. Um valência que influência na velocidade. E mais uma vez o presente estudo vem reforçando e comprovando em média geral que os meninos são mais velozes que as meninas. 
Id on Line Revista Multidisciplinar e de Psicologia

Id on Line Multidisciplinary and Psycology Journal

\section{Conclusão}

Fundamentado no objetivo proposto pelo presente estudo, conclui-se que de forma geral os meninos se saíram melhores que as meninas, quanto à força que eles já possuem geneticamente melhores que as meninas, e isso é uma valência bastante significativa quando se trata de teste de velocidade, e mais uma vez vem a se confirmar o que estudos anteriores já tinham comprovado.

Acredita-se que este presente estudo possa agregar valores importantes e elementos satisfatórios quanto às características da valência física relacionado à velocidade em escolares de 11 a 17 anos de idade e de escolas diferentes, o que poderá colaborar de forma expressiva para o aumento de novos esclarecimentos na área, transformando-se numa nova opção de auxílio a futuras análises sobre o assunto abordado.

\section{Referências}

MARCHESONI, César Costenaro , SALES, Rebeca .A importância da aptidão física relacionada à saúde na escola: EFDeportes.com, Revista Digital. Buenos Aires, Año 15, $\mathrm{N}^{\circ}$ 153 , Febrero de 2011

MELO LMO, Silva MT, Costa IT, Pires FO. Campos CE Relação da flexibilidade na velocidade de corrida de jogadores de futebol: Rev Bras Futebol 2009 Jan-Jun; 02(1): 36-44

OLIVEIRA, Iransé Silva, CRISTINA, Lorena Curado Lopes, PAULA, Rodrigo de Moreira DINIZ, Dênis, RAMALHO, Larissa da Silva. PADRÃO DE VELOCIDADE DOS ESCOLARES DA REDE MUNICIPAL DE ANÁPOLIS: Coleção Pesquisa em Educação Física - Vol.9, n.1, 2010 - ISSN: 1981-4313

PAULO, Luis Gomes Mascarenhas, BISOTTO, Alex Ferreira. Estudo comparativo da aptidão física entre crianças de escola pública e particular: uma visão regional. Revista do Departamento de Educação Física e Saúde e do Mestrado em promoção da Saúde da Universidade de Santa Cruz do Sul / Unisc Ano 14 - Volume 14 - Número 3 - Julho/Setembro 2013 
SANTOS, Edson Farias, ROBERTO, Wellington Gonçalves de Carvalho.Efeito da atividade física programada sobre a aptidão física em escolares adolescentes. Rev Bras Cineantropom Desempenho Hum 2010, 12(2):98-105

\section{Como citar este artigo (Formato ABNT):}

SOUZA, Thiago C.; SANTOS, João D.A.; SOUSA, Paulo G.B. Estudo Comparativo do Nível de velocidade de Jovens de Escolas Públicas de Barbalha e de juazeiro do Norte - CE. Id on Line Revista Multidisciplinar e de Psicologia, Fevereiro de 2017, vol.11, n.34, p. 162-169. ISSN: 1981-1179.

Recebido: 20.02 .2017

Aceito: 27.02 .2017 